\title{
Development of the DVH management software for the biologically-guided evaluation of radiotherapy plan
}

\author{
BoKyong Kim, MD*, Hee Chul Park, MD*, Dongryul Oh, MD, Eun Hyuk Shin, MS, Yong Chan Ahn, MD, \\ Jinsung Kim, PhD, Youngyih Han, PhD \\ Department of Radiation Oncology, Samsung Medical Center, Sungkyunkwan University School of Medicine, Seoul, Korea
}

\begin{abstract}
Purpose: To develop the dose volume histogram (DVH) management software which guides the evaluation of radiotherapy (RT) plan of a new case according to the biological consequences of the DVHs from the previously treated patients.

Materials and Methods: We determined the radiation pneumonitis (RP) as an biological response parameter in order to develop DVH management software. We retrospectively reviewed the medical records of lung cancer patients treated with curative 3-dimensional conformal radiation therapy (3D-CRT). The biological event was defined as RP of the Radiation Therapy Oncology Group (RTOG) grade III or more.

Results: The DVH management software consisted of three parts (pre-existing DVH database, graphical tool, and Pinnacle ${ }^{3}$ script). The pre-existing DVH data were retrieved from 128 patients. RP events were tagged to the specific DVH data through retrospective review of patients' medical records. The graphical tool was developed to present the complication histogram derived from the preexisting database (DVH and RP) and was implemented into the radiation treatment planning (RTP) system, Pinnacle ${ }^{3}$ v8.0 (Phillips Healthcare). The software was designed for the pre-existing database to be updated easily by tagging the specific DVH data with the new incidence of RP events at the time of patients' follow-up.

Conclusion: We developed the DVH management software as an effective tool to incorporate the phenomenological consequences derived from the pre-existing database in the evaluation of a new RT plan. It can be used not only for lung cancer patients but also for the other disease site with different toxicity parameters.
\end{abstract}

Keywords: Radiotherapy, Dose volume histogram, Normal tissue complication probability

\section{Introduction}

Recent advances in the radiation treatment planning (RTP) system, associated with 3-dimensional conformal radiation therapy (3D-CRT) and intensity-modulated radiation therapy (IMRT), now enables us to precisely evaluate and control the dose distribution in the target and critical organs. In order to take full advantage of the state-of-the-art radiotherapy techniques, which promise improved treatment outcomes without increasing the acute and chronic toxicities, dose distribution in the region of interest needs to be accurately controlled in the treatment planning stage. Developing a such treatment plan is, however, challenging because that requires concrete knowledge of the relationship between three dimensional dose distributions and the biological consequences such as tumor control probability (TCP) and

Received 5 March 2012, Revised 22 March 2012, Accepted 26 March 2012.

Correspondence: Youngyih Han, PhD, Department of Radiation Oncology, Samsung Medical Center, 81 Irwon-ro, Gangnamgu, Seoul 135-710, Korea. Tel: +82-2-3410-2604, Fax: +82-2-3410-2619, E-mail: youngyih@skku.edu

*Both authors contributed equally.

(c) This is an Open Access article distributed under the terms of the Creative Commons Attribution Non-Commercial License (http://creativecommons.org/ licenses/by-nc/3.0/) which permits unrestricted non-commercial use, distribution, and reproduction in any medium, provided the original work is properly cited.

www.e-roj.org 
normal tissue complication probability (NTCP).

Many efforts have been made by developing mathematical/ biological models to describe the correlation of the dosimetric parameters and the associated risk of radiation toxicities [1-3]. The prominent models, which describe non-uniform dose distributions in the region of interest, are in reality describing that of uniform dose distribution, specifically to the partial volume of the critical organ [2] or the effective uniform dose to a whole organ [4]. Other models, such as the relative seriality model [3], attempt to derive the partial organ responses from the overall response function of the given organ. All models, however, are confronted with the problem of parameter fitting, which suffers from a lack of good clinical data.

In reality, there are an array of factors, such as various fractionation schedules and different patterns of dose volume histogram (DVH) for a critical organ among different radiation techniques, which could render current models to be too simplistic. Therefore, the model based parameter such as NTCP is still not widely used in many institutions. Although these drawbacks limit the application of proper biological outcome models in the current scene of radiotherapy, eventually, it is necessary to find out solid predictors of biological consequences which can be described by a mathematical or a biological model parameter.

In this regard, it is essential to have a tool that can easily/ effectively handle everyday treatment planning data. We would like to develop the DVH management software which guides the evaluation of radiation therapy (RT) plan of a new case according to the biological consequences of the previously treated cases. Therefore, the software would accumulate the patients' DVH data and the specific DVH data could be tagged according to the incidence of toxicities. The database of DVH and the incidence of toxicities would be updated regularly. It might be used for the prediction of toxicities, evaluation and optimization of treatment planning, and thus, improve the therapeutic ratio of radiation therapy.

\section{Materials and Methods}

To develop DVH management software, we determined the radiation pneumonitis (RP) as an biological response parameter. The incidence of RP was evaluated in the lung cancer patients who were treated with curative 3D-CRT. The biological event was defined as RP of the Radiation Therapy Oncology Group grade III or more.

\section{Patients' characteristics}

In our institute, the treatment policy for stage III non-small cell lung cancer (NSCLC) patients has been concurrent chemoradiotherapy (CCRT). The patients not fit for CCRT because of medical co-morbidities has been treated with highdose radiotherapy alone by $3.0 \mathrm{~Gy} /$ fraction as an alternative measure. Therefore, we had two groups of NSCLC patients treated with different fractionations; the first was of conventional fraction (2.0 Gy/fraction) patients for definitive CCRT and the second was that of high-dose fraction (3.0 Gyl fraction) patients.

We retrospectively reviewed the medical records of lung cancer patients treated with curative 3D-CRT. For the adequate evaluation of radiation pneumonitis, patients who had been followed more than 6 months were included. Among those patients, 128, whose DVH data were retrieved, were enrolled in this study. Sixty nine patients were treated with 3.0 Gy/fraction (range, 45-60 Gy; median, $54 \mathrm{~Gy}$ ) and 59 patients were treated with $2.0 \mathrm{~Gy} /$ fraction (median, $66 \mathrm{~Gy}$ ).

In order to exclude the uncertainties associated with the effect of concurrent chemotherapy on the incidence of RP and the radiobiological parameter $(\alpha / \beta$ ratio), in converting the $3.0 \mathrm{~Gy} /$ fraction to $2.0 \mathrm{~Gy} /$ fraction equivalent dose, we decided the DVH data from each group of patients as a different sets of database. Hence, to find out the cut-off value of RP, we separately analyzed the normal lung DVHs of patients from both treatment groups.

\section{Statical analysis of the patients' data}

For each group, the correlations of the development of RP with the potential predictors were determined using the Cox regression analysis.

The receiver operative characteristics (ROC) curve is a plot of true positive rate against false positive rate. For each dosimetric parameter, ROC area under curve (AUC) value was determined to assess the predictability of a certain parameter regarding the occurrence of $\mathrm{RP}$. The closer the $A U C$ value is to 1.0, the more predictive the DVH parameter is regarding $\mathrm{RP}$ event. A possible association between dosimetric factors was tested with Pearson's correlation coefficient. Factors with a p-value of $\leq 0.05$ were considered to be statistically significant.

The further details of the analysis and findings are fully described at our previous report for the patients treated with 3 Gy/fraction [5]. 


\section{Complication histogram}

The complication histogram was generated according to the methods proposed by Jackson et al. [6]. The DVH management software were developed to guide the evaluation of RT plan of a new case compared to the phenomenological modeling of the pre-existing DVH database of lung cancer patients treated with the same 3D-CRT techniques. The phenomenological models included the graphical representation of individual DVHs tagged with RP events, the cut-off values of dosimetric

Table 1. Clinicial parameters analyzed for correlation with radiation pneumonitis (RTOG grade $\geq$ III)

\begin{tabular}{|c|c|c|c|c|}
\hline \multirow{2}{*}{ Parameters } & \multicolumn{2}{|c|}{$\begin{array}{c}3.0 \text { Gy/fraction } \\
\text { group }\end{array}$} & \multicolumn{2}{|c|}{$\begin{array}{c}2.0 \text { Gy/fraction } \\
\text { group }\end{array}$} \\
\hline & $\begin{array}{l}\text { Hazard } \\
\text { ratio }\end{array}$ & $p$-value & $\begin{array}{c}\text { Hazard } \\
\text { ratio }\end{array}$ & $p$-value \\
\hline \multicolumn{5}{|l|}{ Age (yr) } \\
\hline$<70$ vs. $>70$ & 0.65 & 0.59 & 0.89 & 0.88 \\
\hline Performance & 0.31 & 0.16 & & \\
\hline \multicolumn{5}{|l|}{ Weight loss } \\
\hline No vs. Yes & 0.44 & 0.53 & & \\
\hline \multicolumn{5}{|l|}{ Pre-RT FEV1 (L) } \\
\hline$\geq 2.0$ vs. $<2.0$ & 0.65 & 0.68 & 0.44 & 0.25 \\
\hline Tumor location & 0.43 & 0.45 & & \\
\hline No smoking & & & 0.46 & 0.41 \\
\hline \multicolumn{5}{|l|}{ Total RT dose (Gy) } \\
\hline$\leq 54$ vs. $>54$ & 0.36 & 0.49 & & \\
\hline
\end{tabular}

RTOG, Radiation Therapy Oncology Group; FEV1, forced expiratory volume in 1 second; RT, radiotherapy. factors from ROC analysis, and the complication histogram in the DVH space.

\section{Results}

\section{Analysis of clinical parameters and the DVH parameters} The potential predictors included in the analysis were as follows: clinical parameters (age, performance status, weight loss, pre-RT forced expiratory volume in 1 second [FEV1], tumor location, stage, radiation dose, clinical target volume [CTV]) and the dosimetric parameters (mean lung dose [MLD] and $\mathrm{V} 5-\mathrm{V} 50$ )

On univariate analysis, all these clinical parameters were not significantly associated with the risk of RP (Table 1). However, all dosimetric factors were closely correlated with the development of RP event (Table 2).

\section{Structure of DVH management software}

We designed a software consisted of three parts (pre-existing DVH database, graphical tool, and Pinnacle ${ }^{3}$ script) (Fig. 1). That would be implemented into a RTP system, Pinnacle ${ }^{3}$ v8.0 (Phillips Healthcare, Andover, MA, USA). The software accumulates the patients' DVHs that shall be conveniently sorted according to the tags of RP events evaluated at the time of follow-up for each individual patient.

The first component is a pre-existing DVH database, which were stored with cut-off values of normalized lung volume for the development of RP of grade $\geq I I I$ at every 5 Gy dose steps and the complication histogram. The formation of database

Table 2. Dosimetric parameters analyzed for corelation with radiation pneumonitis

\begin{tabular}{|c|c|c|c|c|c|c|}
\hline \multirow{2}{*}{ Parameters } & \multicolumn{3}{|c|}{ 3.0 Gy/fraction group } & \multicolumn{3}{|c|}{2.0 Gy/fraction group } \\
\hline & Cut-off point (\%) & ROC AUC & p-value & Cut-off point ( $\%)$ & ROC AUC & $p$-value \\
\hline V5 & 60.80 & 0.85 & 0.0001 & 65.90 & 0.74 & 0.0001 \\
\hline V10 & 48.40 & 0.89 & 0.0001 & 57.90 & 0.72 & 0.0001 \\
\hline V15 & 35.10 & 0.89 & 0.0001 & 45.70 & 0.79 & 0.0001 \\
\hline V20 & 27.50 & 0.91 & 0.0001 & 35.10 & 0.78 & 0.0001 \\
\hline V25 & 25.20 & 0.9 & 0.0001 & 28.90 & 0.74 & 0.0001 \\
\hline V30 & 20.90 & 0.9 & 0.0001 & 26.40 & 0.75 & 0.0001 \\
\hline V35 & 18.20 & 0.87 & 0.0001 & 21.20 & 0.8 & 0.0001 \\
\hline V40 & 13.90 & 0.85 & 0.0001 & 18.30 & 0.81 & 0.0001 \\
\hline V45 & 8.80 & 0.82 & 0.0001 & 12.50 & 0.78 & 0.0001 \\
\hline V50 & 3.70 & 0.78 & 0.0008 & 11.80 & 0.75 & 0.0008 \\
\hline MLD & $16.10 \mathrm{~Gy}$ & 0.93 & 0.0001 & $18.94 \mathrm{~Gy}$ & 0.83 & 0.0001 \\
\hline
\end{tabular}

ROC, receiver-operative characteristics; $A \cup C$, area under curve; $M L D$, mean lung dose. 


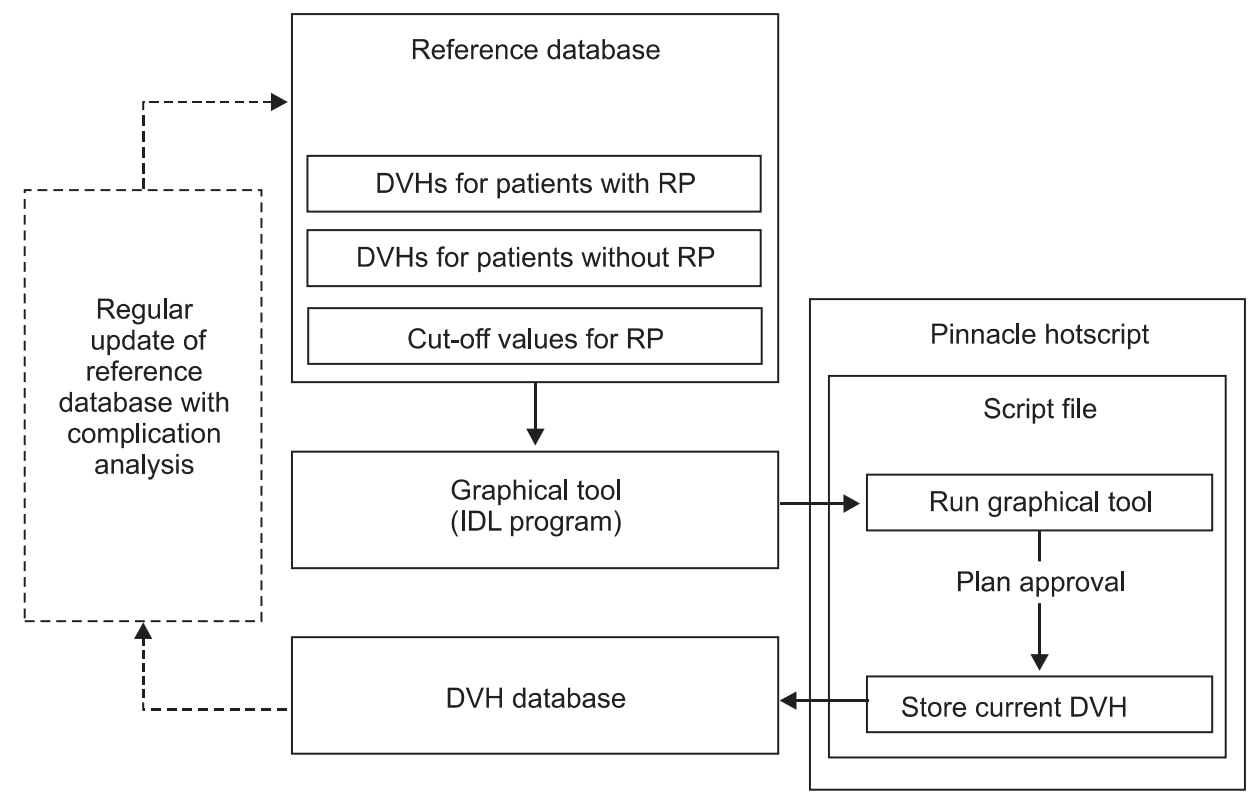

Fig. 1. The structure of the dose volume histogram (DVH) management software. It consists of 3 parts and also needs adding new patient's data and update of the incidence of radiation pneumonitis (RP).

\section{A}

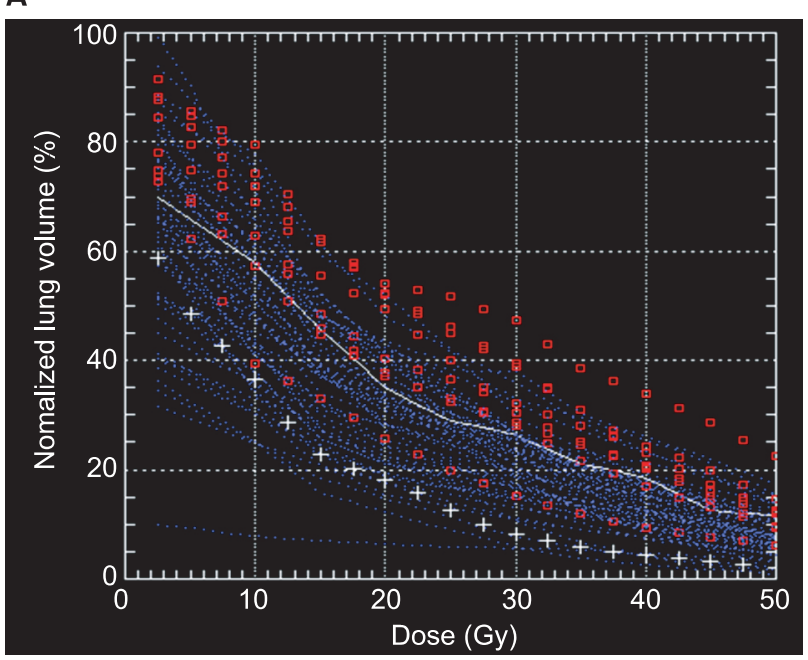

B

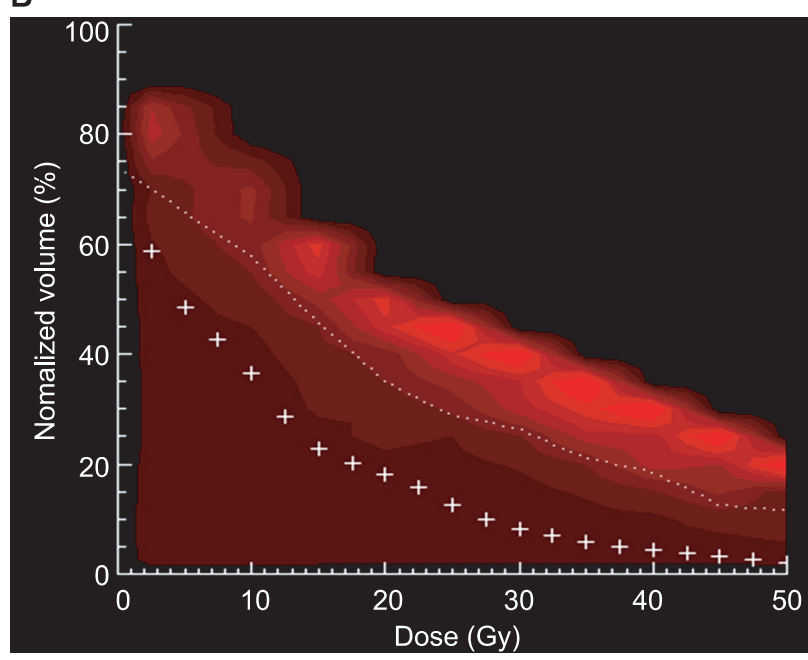

Fig. 2. Graphical plotting of a current dose volume histogram (DVH) data superimposed on the pre-existing DVHs, cut-off values, and the complication histogram represented by graphical tool of DVH management software. (A) Lung DVHs of a new radiation therapy (RT) plan superimposed on the DVHs tagged with RP events and the cut-off values from the receiver operative characteristic (ROC) analysis. Red (blue) color shows DVH data of patients with (without) radiation pneumonitis (RP) event. White solid line indicates the interpolation of the cut-off values and '+' points are DVH data currently evaluted in the new RT plan. (B) Lung DVHs of a new RT plan superimposed on the complication histogram and the cut-off values from the ROC analysis. Atlas of complication histogram is expressed as a color map using the change of brightness. The highier the complication rate is, the color is getting brighter.

with RP event is shortly described in the next section.

The second function is a graphical tool, which enables the superimposition of cumulative normal lung DVH of a new case being evaluated on the pre-existing DVH data. Pre-existing DVHs of patients who suffered from RP are discriminated from those of patients without RP by color on the plot (Fig. $2 \mathrm{~A})$. The complication histogram, which is the incidence of complication on each bin of DVH space, was depicted in color brightness (Fig. 2B). Also, the cut-off values for incidence of RP event were plotted on the same graph to reference each dose step. The graphical tool was coded using a IDL 6.4 (ITT, Boulder, CO, USA). After compiling, the execution file was stored in the same directory where the pre-existing DVH database was located. 
The third component is a Pinnacle ${ }^{3}$ script which provides the graphical tool and stores the DVH database in the planning computer. This script runs the IDL execution files and executes the commend of storing the approved final plan's DVH for a normal lung as a simple text file. The Pinnacle script file was stored in a directory and the 'Hot Script' function provided by the Pinnacle ${ }^{3}$ RTP system was used to run the script. We let the script assigned the name of a DVH file when it was stored, as an identification number of the patient in order to be searched conveniently later on.

\section{Incorporation of the data derived from patients to the software}

The cut-off values of each step of dosimetric factor were defined as the value that shows the highest AUC value from the ROC analysis of each factor. DVH data was separately tagged and sub-grouped according to the incidence of RP, and the data of each sub-group were stored as text files in a designated directory. The atlas of complication histogram was also generated and stored as text files in a designated directory. All these data were incorporated into the newly developed graphical tool which was implemented into the Pinnacle ${ }^{3}$ RTP system.

\section{Clinical use and update of DVH data according to the presence of RP}

When evaluating the RT plan of a new case, physician can refer to the phenomenological modeling from the pre-existing DVH data of other patients. This phenomenological models include the DVHs tagged by the incidence of RP events, the cut-off values from the ROC analysis, and the complication histogram as shown in Fig. 2. The graphical tool runs simply by clicking the button on the 'Hot Script' list of Pinnacle ${ }^{3}$ software. After approving the RT plan, physician or dosimetrist can save the current patient's DVHs by clicking the button on the 'Hot Script' list.

The saved data files should be updated regularly according to the incidence of RP events. The pre-existing DVH data are resorted by the new tags and the cut-off values are reanalyzed with the updated data sets. All the maintenance procedures are easy and simple.

\section{Discussion and Conclusion}

Current RT technologies provides diverse treatment techniques, such as 3D-CRT, IMRT, 4-dimensional radiation therapy, stereotactic radiation therapy, and proton therapy. Many studies have shown different characteristics of DVHs for a certain ROls between the treatment techniques for breast cancer [7], esophageal cancer [8,9] and other disease sites. Moon et al. [7] showed that 3D-CRT, IMRT and proton therapy provided acceptable dose homogeneity in the target of breast cancer, but considerably large volume of low-dose irradiation of heart and ipsilateral lung was observed for patients treated by IMRT. In esophageal cancer radiotherapy, IMRT showed significant reduction of low dose volume of lung [8]. In order to find out the clinical relevance of the observed dosimetric differences as the RT techniques, it is imperative to have the DVHs be tagged and sorted according to the incidence of a certain biological outcome and accumulated systematically.

When the RTP system was upgraded or changed, the recovery of DVHs from the previous system can often be confronted with difficulties. Especially, in the electronic medical record (EMR) environment where paper charts are not available, the importance of having numerical DVH data readable by common desktop software will be important. Our software enables us to store each patient's DVH data systematically. Our software also provides a very useful phenomenological graphical tool. The phenomenological models incorporated in our software can be updated easily. The DVHs can be resorted according to the new incidence of a toxicity parameters. The ROC cut-off values and the complication histogram can be reanalyzed and regenerated easily.

The software developed in the current study can help physicians to evaluate the rival plans for a specific patient from more objective standpoint to estimate the NTCP. And even the physicians can help to score a RT plan even at the premature stage of database development before actively drawing the biological predictors from the accumulated DVH data. The developed software is currently used for the evaluation of RT plans of lung cancer patients in our institution. Therefore, the description pertains to that of lung cancer patients but the same method could be applicable to other disease sites and also other toxicities.

In conclusion, we developed the DVH management software as an effective tool to incorporating the phenomenological consequences derived from the pre-existing database in the evaluation of a new RT plan.

\section{Conflict of Interest}

No potential conflict of interest relevant to this article was 
reported.

\section{Acknowledgments}

This research was supported by the Basic Science Research Program (2011-0005058).

\section{References}

1. Lyman JT. Complication probability as assessed from dosevolume histograms. Radiat Res Suppl 1985;8:S13-9.

2. Kutcher GJ, Burman C. Calculation of complication probability factors for non-uniform normal tissue irradiation: the effective volume method. Int J Radiat Oncol Biol Phys 1989;16:1623-30.

3. Kallman P, Agren A, Brahme A. Tumour and normal tissue responses to fractionated non-uniform dose delivery. Int J Radiat Biol 1992;62:249-62.

4. Niemierko A. Reporting and analyzing dose distributions: a concept of equivalent uniform dose. Med Phys 1997;24:10310.
5. Oh D, Ahn YC, Park HC, Lim DH, Han Y. Prediction of radiation pneumonitis following high-dose thoracic radiation therapy by $3 \mathrm{~Gy} /$ fraction for non-small cell lung cancer: analysis of clinical and dosimetric factors. Jpn J Clin Oncol 2009;39:1517.

6. Jackson A, Yorke ED, Rosenzweig KE. The atlas of complication incidence: a proposal for a new standard for reporting the results of radiotherapy protocols. Semin Radiat Oncol 2006;16:260-8.

7. Moon SH, Shin KH, Kim TH, et al. Dosimetric comparison of four different external beam partial breast irradiation techniques: three-dimensional conformal radiotherapy, intensity-modulated radiotherapy, helical tomotherapy, and proton beam therapy. Radiother Oncol 2009;90:66-73.

8. Chen YJ, Liu A, Han C, et al. Helical tomotherapy for radiotherapy in esophageal cancer: a preferred plan with better conformal target coverage and more homogeneous dose distribution. Med Dosim 2007;32:166-71.

9. Fenkell L, Kaminsky I, Breen S, Huang S, Van Prooijen $M$, Ringash J. Dosimetric comparison of IMRT vs. 3D conformal radiotherapy in the treatment of cancer of the cervical esophagus. Radiother Oncol 2008;89:287-91. 